\title{
Real-Time Market Model for Pricing Differentiated Services
}

\author{
Ruzana Davoyan ${ }^{1,2}$ and Jörn Altmann ${ }^{2,3}$ \\ ${ }^{1}$ Department of Mathematics and Computer Science, University of Mannheim \\ ${ }^{2}$ School of Information Technology, International University in Germany \\ ${ }^{3}$ TEMEP, School of Engineering, Seoul National University, South Korea \\ rdavoyan@mail.uni-mannheim.de,jorn.altmann@acm.org
}

\begin{abstract}
Pricing network resources is a crucial component for proper resource management and the provision of quality of service guarantees. In this paper we propose a decentralized real-time market (RTM) model for pricing differentiated services (DiffServ), which attempts to achieve efficient resource allocation, while reducing computational overheads. We review issues relating to congestion sensitive pricing of the network resources and propose an approach to alleviate price fluctuation that considers the actual state of the network. In comparison with the existing studies that consider the existence of a single point of market equilibrium, we consider the existence of a set of equilibrium states. The proposed strategy relies on the division of link capacity into intervals called steps, where current supply is determined according to the expected resource prices, and is the sum of the allocated steps. Consequently, the real state of the market is described by immediately accessible information, such as price.
\end{abstract}

\section{Introduction}

Since its inception, the Internet has been in a constant state of evolution, driven by an increasing demand for the efficient delivery of bandwidth by a user base, which has expanded both in terms of numbers, and demographics. Modern Internet infrastructure has to allow for the provision of highquality real-time multimedia services, such as videoconferencing, in addition to the best-effort services. In order to achieve a more efficient provision of interactive multimedia content over the Internet, a number of techniques and notions have been formulated relating to the problems of unexpected packet delay, and network latency, such as quality of service (QoS) guarantee. The integrated services
(IntServ) and the differentiated services (DiffServ) frameworks have been standardized by the IETF to aim QoS guarantee. The IntServ architecture [1] is built upon the resource reservation protocol (RSVP) [2] and provides guaranteed QoS. An application that requires end-to-end QoS makes a reservation using the signaling protocol. Each node along a path, using admission control, checks whether sufficient resources are available to accept the new reservation. Unlike IntServ, the DiffServ architecture, specified in [3], classifies traffic according to many different parameters, such as source and/or destination addresses etc., and marks as belonging to a specific traffic class. Traffic classification and marking are done at the edge nodes based on the established between domains service level agreements (SLAs), which specify implicitly or explicitly how transit traffic should be considered when crossing from one domain to another. Within the DiffServ domain each node implements Per-Hop Behaviors (PHBs), which define forwarding properties of a packet associated with a specific class [5], [6]. The PHB is identified by encoding a value in a 6-bit field known as the Differentiated Service Code Point (DSCP) [4], which is stored in the Type of Service (TOS) byte of the IP header, and specifies QoS service semantics. Classification of traffic into a small number of classes reduces flow state information to be kept on each node, and maintenances a simple core, where the decisions of the core nodes are local and based on the determined PHBs. Because of its simplicity and scalability, the DiffServ architecture is foreseen to be implemented in the Internet [7]. Differentiation of the Internet services in respect to QoS has to rely on pricing in order to allow the users to choose appropriate services. Unlike pricing IntServ, which is based on allocated resources, pricing DiffServ is more complicated and at present is an open issue. Pricing plays a fundamental role to convey information about resource availability among networks and to control network congestion. 
There exists a large body of literature regarding pricing approaches, which are mainly focused on setting optimal prices based on computation of marginal congestion costs. While achieving the social welfare or provider's revenue maximization [14] under detailed calculation of marginal congestion costs, dynamic pricing leads to undesirable for the user price fluctuation and computational overheads required to derive these prices. The main goal of our research was to introduce economic model for efficient resource management, which alleviates complexity of the dynamic pricing strategies. This study can be differentiated from the others in two ways. Firstly, the main idea behind the proposed strategy of reducing price fluctuation is approximation of average marginal congestion costs, under the division of link capacity into intervals, referred to as steps. Therefore, in comparison with the existing approaches, which consider utilization of the full link, while setting optimal prices, we consider capacity as a dynamically changing function, which is based on market expectations. And secondly, movement of a whole system is described by a two-period economy [24], [28], where providers, under certain circumstances form expectations about future spot prices, and then make decisions about supply plans in order to eliminate the existence of equilibrium perturbation in the system. It is important to note, that since the realtime market state is described by immediately accessible information, such as price, in our model provider's expectations about future spot price are based on a spot price observation, although history of supply and demand is also available.

The work in [8] is related to ours in a sense of interpreted supply function. However, these two approaches differ significantly. While we assume that supply is a sum of allocated steps, i.e., it is not of necessity that all allocated resources are used the approach in [8] considers supply as the actually supplied capacity. Besides, we reduce price fluctuation by considering system floating between the equilibrium states. Considering dynamic demand, in our approach there are few equilibrium points corresponding to an offered supply, while in [8] there is one equilibrium point for an offered load. Finally, the proposed pricing strategy never tends to infinity, because this causes a link to be idle. Proposed in the work [9] expected capacity pricing approach is also focused on users' expectation. In comparison with [9], where the customers are charged based on the expected volume of traffic, in this paper the users are charged based on an actual usage, according to the spot market price. Whereas the main idea of the edge pricing approach [10] is charging the customers based on the expected values of congestion and route, assuming that the utility and demand functions are stable over time, current study considers these characteristics dynamic and provides the pricing strategy based on the actual network state.

This paper is organized as follows. Section 2 describes background focusing on various issues of pricing network resources. Section 3 describes the proposed real-time market (RTM) model with capacity division approach and finally, Section 4 concludes the paper.

\section{Background and Fundamental Issues}

\subsection{Optimization Problem}

Pricing network resources has drawn considerable attention in research area [9]-[12], [19]. A detailed overview and evaluation according to the different criteria of the proposed pricing schemes is given in [13]. Most of the studies seek to achieve economic (Pareto) efficiency [14], by establishing an optimization model and finding an optimal price for the network resource allocation. An optimization problem can be formulated as follows [14].

Consider the market with a set of consumers $N=\{1, \ldots \ldots n\}$ and services $m$. Let $x^{i}$ is the amount of services consumed by customer $i \quad(i \in N)$ and $x=x^{1}+\ldots+x^{n}$ denote the amount of services given to the users from 1 to $n$ correspondingly, i.e. the total demand. The utility function $U^{i}\left(x^{i}\right)$ is equivalent to a certain monetary value, that customer $i$ is willing to pay having a vector of quantities of $m$ services $x^{i}=\left(x_{1}^{i}, \ldots, x_{m}^{i}\right)$. The consumer surplus maximization problem is defined as follows:

$$
\begin{gathered}
\qquad S_{i}=\max _{x^{i}}\left[U^{i}\left(x^{i}\right)-p^{i}\left(x^{i}\right)\right], \\
\text { s. t.: a set of constraints, e.g. } p^{i}\left(x^{i}\right) \leq b^{i}
\end{gathered}
$$

for all $i \in N$, where $p^{i}\left(x^{i}\right)$ represents the actual amount that consumer $i$ pays for the bundle $x^{i} ; b^{i}$ is a budget constraint of customer $i$. On the other hand, the provider tries to maximize own revenue by solving the following problem:

$$
\max _{x}[R(x)-C(x)]
$$

where $R(x)$ denote revenue gained by the provider selling the network resources $x ; C(x)$ is cost of service provider producing network resources $x$. Thus, optimization problem of the whole system is 
defined as a sum of all utilities (social welfare) maximization and is described as follows:

$$
\max _{x^{i}} \sum_{i} U^{i}\left(x^{i}\right), \quad \text { s. t. : } \sum_{i} x^{i} \leq x
$$

In essence, the existing approaches differ in a way of computing $U^{i}\left(x^{i}\right), R(x)$, and $C(x)$, as well as considering different set of constraints. In order to maximize social welfare (3) or provider's revenue (2) the proposed pricing schemes [9]-[12] assume a priori knowledge of either a well-known user utility function or a demand function (based on market research) accordingly. A utility function, which expresses user's willingness to pay for a certain QoS guarantee, in some approaches depends on the QoS metrics, such as delay or packet loss [12], [15], [16]. In real networks, however, it is impossible to predict such quality measures. As an application-layer performance measure, a utility function is quantified as an amount of the network resources allocated to a flow. Therefore, it is meaningful to distinguish elastic and inelastic traffic [17]. Unlike elastic traffic, which is tolerant to available bandwidth variation, inelastic traffic requires a fixed amount of bandwidth for a particular QoS. However, in [10] was argued that a utility function cannot be defined precisely in shortterm and sometimes even in long-term time frames, and in addition, user demand is difficult to predict. Thus, whether optimality is achieved in real world is still under doubt. Besides, the study in [10] argued that pricing network resources should shift away from the optimality paradigm and attempt to achieve reasonable level of efficiency.

\subsection{Static vs. Dynamic Pricing}

Pricing approaches that assume a priori knowledge of a demand function can be differentiated according to the time frames criteria. As a consequence, pricing schemes are classified into long (day or more) and short (minutes or less) pricing time frames, called static and dynamic pricing accordingly. Regarded as static pricing, usage-based charges [14] in the Internet can be two types: access time and total data volume charges. Another form of static pricing is flat-rate [14] under which the users are charged a monthly subscription regardless of usage and actual network conditions. Therefore, flat-rate pricing gives no incentive to the users to react on congestion or to limit their usage. Long pricing time frames, which consider a demand function stable over a contract period of time, do not address short-term congestion issue. Actually time-of-day is not able to preserve system equilibrium in terms of supply-demand balance, since a price is independent of the current network load.

On the other hand, a dynamic price [14] depends on current network utilization, and is flexible to control congestion. Although the pricing schemes that focus to set price over a short period of time, maximize economic efficiency, deployment of such complex schemes is unrealistic. Whereas proposing dynamic pricing plans, it is possible to provide better QoS, such complex prices are unacceptable by users who are risk averse. Indeed, the customers do not want to face with varying prices, which may be difficult to understand. An experimental characterization of users' behavior in relation to the prices of the network services is provided by the INDEX project [21], [22]. Besides, it was argued in [10], that price fluctuation causes high implementation costs and structural requirements.

\subsection{Congestion Impact}

Congestion is an example of negative market externality when a user can make different choices, which affect the other users, without suffering penalty [18]. Designed congestion costs address this issue. By charging the users, the provider tries to cover part or all costs (e.g. five types of costs as suggested in [19]) that occur in the computer networks. Congestion pricing scheme reflects on the actual state of the network in terms of excess demand for the network resources and covers congestion costs that a user imposes to the other users. During congestion the users are expected to adjust their sending rate [23], [11] in response to explicit (i.e. response from a congested router) or implicit (i.e. based on externally observable behavior of the network) feedbacks. However, it has been shown that although, sometimes congestion periods have a few milliseconds duration, and the users cannot react instantaneously, such deviations do not lead to network instability [20].

While solving the optimization problem most of the studies set prices at exactly the marginal congestion costs. When there are available resources in the network, i.e. demand does not exceed capacity, marginal congestion cost is essentially zero. The study in [10] has criticized the optimality paradigm by posing three reasons. The first argument is that marginal costs are small and insufficient to fully recover facility costs. In addition, it was argued that these costs are irrelevant to the efficiency of a system as a whole. Second reason shows inaccessibility of the marginal congestion costs. Indeed, since impact of congestion on different applications differs, the pricing schemes should take into account tolerance and intolerance of an application towards variation of packet delay and drop. As a consequence, it is hard to 
measure the exact contribution of a particular flow to congestion. The last fact is that besides optimality, structural and implementation issues should be considered for practicability. Therefore, it is not the goal to reach optimality, but enough to set a price that provides an appropriate QoS level required for an application.

\subsection{Economic Equilibrium}

The basic principle of economic equilibrium requires knowledge of supply and demand functions, and defines a competitive market price [25]. Indeed, if both supply and demand are the functions of quantity and price, and are known, then the market price can be defined for a particular quantity of the product. While economic forces are balanced in the absence of external influences, this price is an equilibrium price. Walrasian equilibrium, aka competitive equilibrium, assumes that there is no excess in demand for any good when each participant buys the bundle, which is optimal for him under own budget constraint [14]. In general, the theoretical solution of general equilibrium describes the "static" equilibrium conditions, i.e. market clearing equations. Unlike the theoretical, the practical solution of a system describes a "dynamic" adjustment process, called tâtonnement process [14], i.e. Walrasian price-adjustment rule, which implies that no transaction and no production take place at price disequilibrium. If good demand excess, then price increases, and decreases for the goods with excess supply. Setting optimal price assumes that customer's utility function depends only on the commodities that she or he consumes. However, in real world, there is positive economic externality, when value of a unit increases, as more users use it [18].

As cited in [26], "in reality, the equilibrium is never reached since, in so far as one tries to approach it, it continuously changes as the technical and economic conditions of production change. The real state is then a state of persistent oscillations around a central equilibrium point, which itself drifts away". In spite of useful economic arguments for competitive equilibrium, in real life competitive equilibrium is often inapplicable of providing a practical means of pricing. The reason of such concept is that instead of observing total demand or total supply in real market, the prices of traded commodities are observed. Statistical analysis of historical data can be used; however, real-time state of the market is described by the prices. Besides, taking into account dynamic behavior of the whole system, it is meaningful to consider a set of equilibrium states, rather than one point of system equilibrium.

\section{Real-Time Market Model}

\subsection{Motivation of Dynamic Equilibrium}

The basic idea of a market model is that system deviation from the equilibrium state produce forces, which try to return it to the equilibrium point as depicted in Fig. 1. Point E in Fig. 1 is an equilibrium state of a system, where the market clears price $\mathrm{P}_{0}$, under equal supply $\mathrm{S}$ and demand $\mathrm{D}$. This equilibrium notion is similar to one of the necessary conditions of mechanical equilibrium, stating that the sum of forces on each particle of a system is equal to zero. If the price is set higher than the equilibrium one $\left(\mathrm{P}_{1}\right.$ in Fig. 1 ), it causes to surplus, i.e. supply exceeds demand. As a result, to sell this surplus provider decreases price. This encourages demand, and eventually equilibrium point will be reached. The opposite would happen if price were lower than equilibrium one $\left(\mathrm{P}_{2}\right.$ in Fig. 1).

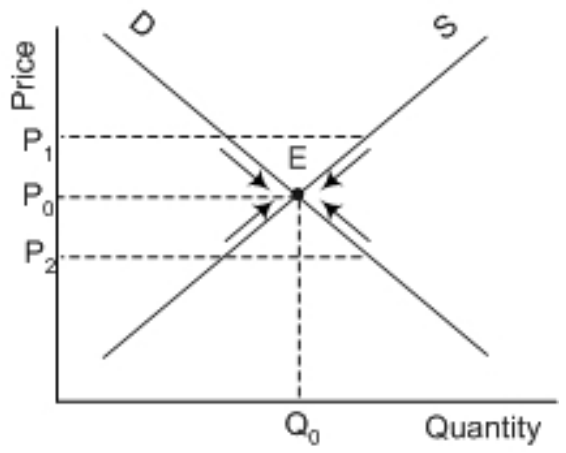

Figure 1. Market mechanism: the market clears at price $P_{0}$ and quantity $Q_{0}$.

However, taking into account that the system components, such as users' preferences and supply change over time, and the whole system is driven by a general movement we consider not the existence of a singular point of a solution, but rather movement along equilibrium. Thus, instead of setting optimal price we adopt the notion of dynamic equilibrium, where the market system consisting of the independent dynamic subsystems, such as supply and demand is in equilibrium, when all subsystems are in equilibrium in respect to each other. As a consequence of dynamic nature of the market, we assume that there should be not just a single equilibrium point, but a set of equilibrium states of the system. In addition to this, instead of considering the market that moves along equilibrium, as a set of price adjustment processes, where the price is regulated based on demand and supply, it is more meaningful to consider the market, as a set of market clearing processes [25], where the 
market will eventually be cleared of all supply and demand excesses.

\subsection{Capacity Division}

As mentioned before, most of the literature is based on setting optimal prices so that all resources are utilized, while achieving social welfare or provider's profit maximization. Since the current paper is focused to reduce undesirable price fluctuation, considering incremental congestion costs, we do not intend to provide optimal price, but attempt to achieve reasonable efficiency of the whole system. Thus in comparison with the existing approaches, which consider total link capacity, while optimizing resource allocation, we divide total capacity into intervals called steps, in order to control link utilization. In other words, a step indicates a certain level of link utilization. Therefore, the supply function is defined not by the total link capacity, but by a sum of allocated steps. We assume that within each step average costs as well as average marginal congestion costs remain unchanged. Indeed, considering U-shape average cost curve, average costs for production of different amount of the goods may be the same [25]. The motivation of the assumption of equal average marginal congestion costs is that congestion characteristics, such as utilization, queue length etc. are not instantaneous values, instead they are average measurements. Based on these prerequisites, we claim, that average resource allocation costs for a particular step of a certain link are unchanged. Steps can be chosen by network administrator for all links within a domain and made known to its routers. Empirical values or management policies could be used in order to set values of the steps.

Taking into account that average allocation costs remain unchanged for each step, price defined by the provider for each step, called step price is unchanged within an interval, associated with a certain step. Consequently, neutral equilibrium is considered along step, meaning that the step price is constant for the allocated step when dynamically changing demand lies within the step bounds. A certain step price covers all costs appeared in the network for corresponding step. In order to maintain different QoS levels for the DiffServ architecture, appropriate step prices for the certain QoS levels should be defined by the provider.

\subsection{Two-period Model}

Since the real state of the market is described by immediately accessible information, such as a price, we adopt a two-period economy notion [24][28], in order to describe how the state of the dynamic market is changed. At first, the provider observes the spot price, forms expectation about future spot price, and then makes decision about supply plans in order to eliminate market disequilibrium. More specifically, the notion of a two-period economy is described as follows. Traders observe the spot price, and make expectation about a price in the next period. Generally, price deviation is not revealed to the observer instantaneously. In other words, price fluctuation does not reflect on demand immediately and the reason of such deviation is not clear for the buyers. At the same time the suppliers make assumption about the future, whether the price continues to increase or decrease. In case the suppliers decide that the price continues to increase, production, i.e. sum of the allocated steps, will be increased in order to balance the market. Consequently, this encourages demand and as a result, the market state is defined by feedback from the suppliers, i.e. how they react on price change. Eventually, supply decisions are based on the expected prices of the resources. Expectations of the traders about the future market state depend on the current market circumstances and the statistical analysis of historical data. However, it is beyond the scope of this paper to concern traders' expectations.

We model a system as the market, where the provider observes the spot price, and defines some expectation about the future spot price and the state of a system. Thus, we assume that a current supply decision is regulated by the expected resource price.

Let us assume that the current price is $p$ with a certain confidence that the expected price will be $p_{\text {exp }}$, and is defined as follows:

$$
p=\frac{p_{\text {exp }}}{1+R}
$$

where $R$ is the rate of return. However, in a decentralized environment ex-ante supply does not match ex-ante demand instantaneously, i.e. the system cannot immediately come to an equilibrium. Therefore, within the first period the spot price adjusts in proportion to the current ex-ante excess or shortage demand according to the following adjustment rule:

$$
\dot{p}=k E
$$

where $k>0$ is a constant that is determined through a qualitative analysis of the dynamics of the market price, and $E$ denotes the current ex-ante excess or shortage demand function that is calculated by the following equation:

$$
E=D(p)-S
$$

where $D(p)$ is the total ex-ante demand and $S$ 
represents the total ex-ante supply of resources. Exante excess or shortage demand can take on positive and negative values, meaning price increase and decrease accordingly. In the proposed approach, the following cases can be considered: a) the spot price increases and adjusts according to the rule (5) if $D(p)>S, \mathrm{~b})$ the spot price is set to the appropriate step price of the last allocated step, if the ex-ante demand lies within the last allocated step bounds, i.e. $S-x_{i} \leq D(p) \leq S$, where $x_{i}$ is the size of the last allocated step, c) otherwise, the spot price decreases and adjusts according to the rule (5). Therefore, the spot price is either set to the respective step price or adjusts according to the price adjustment rule. It is worth noting, that price adjustment rule does not represent the tâtonnement process, and differs in sense of market clearing. While the tâtonnement process always implies price rationing (e.g. price will decrease if supply exceeds demand), adjustment rule can involve quantity rationing (price can remain unchanged if supply exceeds demand). The total exante supply of resource adjusts according to the following rule:

$$
\dot{S}=\left\{\begin{array}{cccc}
\neq 0 & \text { if } & p \neq \frac{p_{\text {exp }}}{1+R} \\
0 & \text { if } & p=\frac{p_{\text {exp }}}{1+R}
\end{array}\right.
$$

Thus, the rule (7) shows that if the spot price does not match the expected one, providers review their plans and make further supply decisions. Fig. 2 illustrates an example of the above-described mechanism. The D1 and D2 lines represent the maximal ex-ante demand for the appropriate allocated steps, such as $\mathrm{Q}_{0}$ and $\left(Q_{o}, Q_{2}\right]$, with the step prices $\mathrm{P}_{0}$ and $\mathrm{P}_{2}$. Let us assume that the step $\mathrm{Q}_{0}$ is allocated and the user is charged the price $\mathrm{P}_{0}$, while demand does not excess supply ( $\mathrm{P}_{0} \mathrm{~A}$ interval). If demand exceeds upper bound of the allocated step $\mathrm{Q}_{0}$, the spot price increases and adjusts according to the rule (5) (AB interval) with ondemand allocation of the $\left(Q_{o}, Q_{1}\right]$ resources. It is important to note, that the provider does not allocate the step $\left(Q_{o}, Q_{2}\right]$ immediately, but rather on-demand resource allocation is performed under the adjusted spot price. Once the spot price $\mathrm{P}_{1}$ is higher than the expected one $\mathrm{P}_{2}$, implies future excess demand, and the provide increases supply on the step at the end of the point $\mathrm{Q}_{2}$ in Fig.2, i.e. the supply curve shifts to right. Increase in supply when the future demand exceeds, switches the price to the step price of the last allocated step, i.e. to $\mathrm{P}_{2}$. If price were lower than expected one, the opposite would happen.

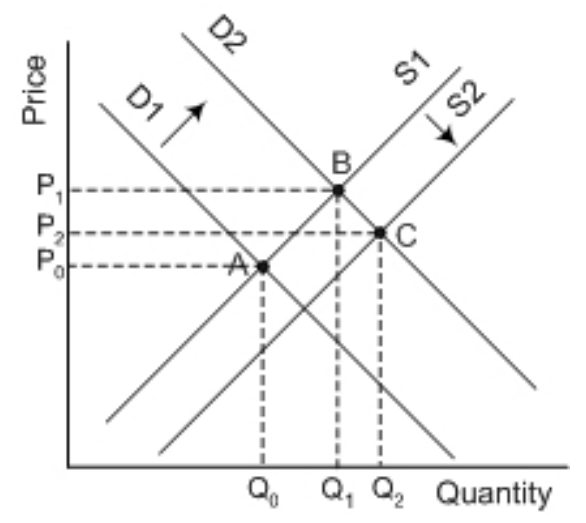

Figure 2. Supply allocation.

Taking into account that within each step neutral equilibrium is proposed, however, the whole system is described by dynamic equilibrium the RTM model is presented as a set of market clearing processes (i.e. the market clears at the appropriate step price under the allocated supply). Abstracting each step interval into a single equilibrium point, since the price is unchanged within the allocated step, whereas dynamic demand does not exceed step bounds, we can assume a set of equilibrium points. Economic stability [27], considers that the system is stable if demand curve is almost always represented as downward sloping, meaning that as price decreases, customer will buy more of the goods, and supply is upward sloping, meaning that as price increases, supplier wish to produce more of the goods. In essence, if the spot price adjusted according to the price adjustment rule (5) is less than the expected price, this encourages demand and consequently, switches the price to the step one and vice versa.

Another feature of the proposed approach is that price never reaches infinity. Generally under high load of the network, the prices of the resources are too high and tend to infinity. In this case all traffic will move off the link, advertised high price. As a consequence, the link will stay idle until it would advertise a low price. The effect of this instability is that under heavy load, many links would be idle, which is not desired in practice. Instead, increase in price is likely to be unattractive to some paths or services, but can still be attractive and remain the best choice for other services.

\section{Conclusions}

In this paper we presented the decentralized realtime market (RTM) model for pricing in DiffServ 
networks, which alleviates complexity of congestion based pricing, and achieves efficient resource allocation. We have reviewed the issues relating to dynamic pricing of the network resources, and proposed the strategy to significantly reduce price fluctuation and computational overheads. For the purpose of alleviating price variation, we have divided link capacity into the steps, with approximate average marginal congestion costs, and have considered neutral equilibrium within each step. The proposed pricing strategy relies on the actual state of a system, and reflects on available in the network resources, allocated in real time. In addition, the approach is simple and scalable, and allows network administrators to make their own decision about how to set prices.

The state of the RTM model is described by the prices of the traded resources, where the providers make decision on the current supply based on the expected prices. Considering the dynamical behavior of users' preferences and supply, the movement of the whole system is described by a set of equilibrium points, each of which represents the step abstracted into a single equilibrium point. Thus, the proposed model is treated as a dynamic system composed of the subsystems, such as supply and demand, which are in equilibrium relative to each other.

\section{References}

[1] R. Braden, D. Clark, and S. Shenker, "Integrated services in the Internet architecture: An overview", IETF RFC 1633, June 1994.

[2] R. Braden, L. Zhang, S. Berson, S. Jamin, "Resource reservation protocol (RSVP) - version 1 functional specification", IETF RFC 2205, Sept. 1997.

[3] S. Blake, D. Black, M. Carlson, E. Davies, Z. Wang, W. Weiss, "An architecture for differentiated services", IETF RFC 2475, Dec. 1998.

[4] K. Nichols, S. Blake, F. Baker, D. Black, "Definition of the differentiated services field (DS Field) in the IPv4 and IPv6 headers", IETF RFC 2474, December 1998.

[5] V. Jacobson, K. Nichols, K. Poduri, "An expedited forwarding PHB", IETF RFC 2598, June 1999.

[6] J. Heinanen, F. Baker, W. Weiss, J. Wroclawski, "Assured forwarding PHB group", IETF RFC 2597, June 1999.

[7] K. Kilkki, "Differentiated services for the Internet", Macmillan Technical Publishing, 1999.

[8] T. Li, Y. Iraqi, R. Boutaba, "Pricing and admission control for QoS-enabled Internet", Computer Networks, Vol. 46, no.1, 87-110, 2004.

[9] D. Clark, "Internet cost allocation and pricing", in Internet Economics, L. W. McKnight and J. P. Bailey, Eds., MIT Press, pp. 215-252, 1997.

[10] S. Shenker, D. Clark, D. Estrin, S. Herzog, "Pricing in computer networks: reshaping the research agenda",
ACM Computer Communications Review, pp. 19-43, 1996.

[11] X.Wang, H. Schulzrinne, "Pricing network resources for adaptive applications", IEEE/ACM Transactions on Networking, 14(3): 506-519, June 2006.

[12] A. Odlyzko, "A modest proposal for preventing Internet congestion", Technical report TR 97.35.1, AT\&T Research, Florham Park, New Jersey, Sept. 1997.

[13] M. Falkner, M. Devetsikiotis, I. Lambadaris, "An overview of pricing concepts for broadband IP networks", IEEE Communications Surveys, Vol. 3, no. 2. 2nd quarter 2000.

[14] C. Courcoubetis, R. Weber, "Pricing communication networks: economics, technology and modeling", Wiley, March 2003.

[15] R. Cocchi, D. Estrin, S. Shenker, L. Zhang, "Pricing in computer networks: motivation, formulation, and example, IEEE/ACM Transactions on Networking, Vol. 1, no 6, pp. 614-627, Dec. 1993.

[16] L. DaSilva, D. Petr, N. Akar, "Equilibrium pricing in multi-service priority-based networks", in Proc. IEEE GLOBECOM, Phoenix, AZ, pp. S38 6.1-6.5, Nov. 1997.

[17] S. Shenker, "Fundamental design issues for the future Internet", IEEE Journal of Selected areas in Communication, Vol. 13, no. 7, pp. 1141-1149, Sep. 1995.

[18] N. Economides, "The economics of networks", International Journal of Industrial Organization, Vol. 14, no. 6, pp. 673-699, October 1996.

[19] J. Mackie-Mason and H. Varian, "Pricing the Internet", Public Access to the Internet, Brian Kahin and James Keller eds., Cambridge, Mass.: MIT Press, pp. 269-314, 1995.

[20] F. Kelly, "Models for a self-managed Internet", Philosophical. Transactions of the Royal Society, Vol. 358, pp. 2335-2348, 2000.

[21] J. Altmann, B. Rupp, V. Pravin, "Effects of pricing on Internet user behavior", NETNOMICS, Volume 3, Issue 1, pp. $67-84$, Jun 2001.

[22] J. Altmann, K. Chu, "A proposal for a flexible service plan that is attractive to users and Internet service providers", IEEE INFOCOM, Anchorage, USA, April 2001.

[23] J. MacKie-Mason, L. Murphy, J. Murphy, "Responsive pricing in the Internet". In Bailey, J. P. \& McKnight, L. W. (eds) Internet Economics. Massachusetts: MIT Press, pp. 279-303, 1997.

[24] Y. Stournaras, "On the stability of the short-run equilibrium of an exhaustible resource market", Oxford Institute for Energy Studies Staff Softcover, 1984.

[25] R. Pindyck, D. Rubinfeld, "Microeconomics", Prentice Hall, Sixth Edition, 2005.

[26] F. Donzelli, "The epistemological status of general equilibrium theory: a historical perspective", $3 \mathrm{rd} \mathrm{PhD}$ Conference in Economics, Sep. 2005.

[27] H. Varian, "Microeconomic analysis", Third Edition, WW Norton and Company, 1992.

[28] E. Schlicht, "Isolation and Aggregation in Economics", Springer-Verlag: Berlin-Heidelberg-New York 1985. 